\title{
The Role of Prostaglandins and Endothelium- Derived Relaxation Factor in the Regulation of Cerebral Blood Flow and Cerebral Oxygen Utilization in the Piglet: Operationalizing the Concept of an Essential Circulation
}

\author{
WILLIAM MEADOW, BRIAN RUDINSKY, ANTHONY BELL, MARIE LOZON, CHESTER RANDLE, \\ AND ROBERT HIPPS
}

Department of Pediatrics, The University of Chicago, Chicago, Illinois 60637

\begin{abstract}
The brain is considered an "essential" organ, defined as one whose blood supply is preferentially maintained vis-à-vis other less-essential circulations during periods of reduced systemic cardiac output (CO). We asked whether the actions of either prostaglandins or endothelium-derived relaxation factor might underlie the essential qualities of the cerebral circulation; that is, would the absence of one or the other impair the ability of the brain to preferentially redirect systemic blood flow during a period of reduced systemic $\mathrm{CO}$. We compared hemodynamics in the cerebral and systemic circulations in $\mathbf{3 3}$ anesthetized piglets under three conditions that reduced systemic $\mathrm{CO}$ equivalently: endothelium-derived relaxation factor inhibition with the substituted $\mathrm{L}$-arginine analog $\mathrm{N}$ nitro-L-arginine (NNLA; $25 \mathrm{mg} / \mathrm{kg}$ ), prostaglandin inhibition with indomethacin (INDO; $5 \mathrm{mg} / \mathrm{kg}$ ), and inflation of a left atrial balloon (LAB) catheter. NNLA, INDO, and $\mathrm{LAB}$ each reduced $\mathrm{CO}$ to an equivalent value $(\sim 30 \%$ from baseline). NNLA and INDO, but not LAB elevated systemic blood pressure, cerebral perfusion pressure (CPP), systemic vascular resistance (SVR), and cerebral vascular resistance (CVR). Cerebral blood flow (CBF) was preserved after NNLA and LAB but fell after INDO ( $-35 \%)$. Despite the equivalent reduction in $\mathrm{CO}$ noted during the three experimental protocols, the proportion of systemic blood flow directed toward the brain (CBF/CO) rose significantly during LAB and NNLA $(+30 \%)$ but fell significantly during INDO $(-12 \%)$. Similarly, relative cerebral vascular resistance (CVR/SVR) fell significantly during LAB and NNLA but rose during INDO. Cerebral vascular regulation can be considered along two complementary dimensions. Vascular regulation within the cerebral circulation itself (cerebral autoregulation) is expressed as CBF versus CPP. CBF was unchanged as CPP fell during $\mathrm{LAB}$ and as CPP rose after NNLA in piglets. In contrast, after INDO, CBF fell as CPP rose. Vascular regulation of the cerebral circulation vis-à-vis the rest of the body (referred to here as cerebral-specific vascular regulation) can be expressed either in terms of blood flow (CBF/CO) or vascular resistance (CVR/SVR). After both NNLA and $\mathrm{LAB}$, as CO fell, CBF/CO rose and CVR/SVR fell, demonstrating preservation of the "essential" status of the cerebral circulation. In contrast, after INDO as systemic CO fell, CBF/CO fell and CVR/SVR rose. Prostaglandins,
\end{abstract}

Received June 24, 1993; accepted January 19, 1994

Correspondence: William Meadow, M.D., Ph.D., Department of Pediatrics, MC 6060, Wyler Children's Hospital, 5825 S. Maryland Ave., Chicago, IL 60637.

Supported in part by the Children's Research Foundation of Chicago. but not endothelium-derived relaxation factor seem critical both for cerebral autoregulation and for preservation of the essential hemodynamic status of the brain vis-à-vis the rest of the body in piglets. (Pediatr Res 35: 649-656, 1994)

Abbreviations

ANOVA, analysis of variance

BP, blood pressure

CBF, cerebral blood flow

$\mathrm{CO}$, cardiac output

CPP, cerebral perfusion pressure

CVO2, cerebral oxygen consumption

CVR, cerebral vascular resistance

EDRF, endothelium-derived relaxation factor

ICBF, internal carotid artery blood flow

INDO, indomethacin

LA, left atrium

LAB, left atrial balloon

NNLA, N-nitro-L-arginine

NO, nitric oxide

PA, pulmonary artery

PG, prostaglandin

QO2, systemic oxygen delivery

RA, right atrium

SS, sagittal sinus

SSP, sagittal sinus pressure

SVR, systemic vascular resistance

VO2, systemic oxygen consumption

The brain is considered an "essential" organ, defined as one whose blood supply is preferentially maintained during periods of reduced $\mathrm{CO}$ and $\mathrm{QO} 2$. This preferential perfusion of the brain may occur even at the expense of other less-essential circulations (e.g. renal, mesenteric). The mechanisms underlying the ability of the cerebral circulation to maintain its preferred status vis-àvis the systemic circulation are not well understood.

Two important classes of paracrine mediators that have been postulated to regulate various aspects of cerebral perfusion are PG and EDRF (1-8). We asked whether the actions of either of these two categories of vasoactive agents might underlie the "essential" quality of the cerebral circulation in the piglet; that is, would the absence of one or the other impair the ability of the brain to preferentially redirect or use systemic blood flow during a period of reduced $\mathrm{CO}$ and $\mathrm{QO} 2$. 
To address this question, in piglets we compared hemodynamics and oxygen utilization in the cerebral and systemic circulations in piglets under three conditions that reduced $\mathrm{CO}$ and QO2 equivalently: PG inhibition with INDO, EDRF inhibition with the substituted L-arginine analog NNLA, and inflation of a LAB catheter.

\section{MATERIALS AND METHODS}

Anesthesia and Ventilation. Piglets (1-2 wk old; $n=33)$ received ketamine intraperitoneally $(20 \mathrm{mg} / \mathrm{kg})$ and were intubated endotracheally. An ear vein was cannulated for venous access. The piglets were anesthetized with sodium pentobarbital $(20 \mathrm{mg} / \mathrm{kg}$ i.v.), and muscle relaxation was achieved with $d$ tubocurarine $(1 \mathrm{mg} / \mathrm{kg})$. Throughout the experiment, the level of anesthesia was assessed by determining the response of heart rate and BP to noxious stimuli and adjusted by intermittent bolus of pentobarbital $(\sim 2 \mathrm{mg} / \mathrm{kg} / \mathrm{h})$. Mechanical ventilation (Harvard Small Animal Ventilator, Harvard Medical Supplies, Dover, MA) was used, with the following initial ventilator settings: fraction of inspired oxygen $=0.30$, positive end expiratory pressure $=3 \mathrm{~cm} \mathrm{H} \mathrm{O}$, tidal volume $=15 \mathrm{~mL} / \mathrm{kg}$, and rate $=10$ breaths/min. Warming blankets and overhead heating lamps were used to maintain body core temperature at $37-38^{\circ} \mathrm{C}$. A femoral artery catheter was placed to provide access to arterial blood and monitor systemic BP. A suprapubic cystostomy catheter was placed to establish urinary drainage.

Thoracic Surgery. Polyethylene catheters were surgically introduced into the RA (via left external jugular vein) and into the PA and LA (via left lateral thoracotomy). In the piglets receiving the LAB protocol (see below), a balloon catheter (3-mL balloon volume) was placed in the LA instead of the polyethylene catheter. Following our previously described methodology $(9,10)$, an external electromagnetic flow probe (EP 425; Carolina Medical Electronics, King, NC) was placed around the PA, proximal to the tip of the PA catheter. During each experiment, the PA flow probe was zeroed frequently using the approximation that diastolic blood flow in the PA is zero. This "diastolic zero" assumption has been shown to be appropriate both when PA pressure is normal and during periods of elevated PA pressure (such as occurred during NNLA infusion; see below). In the documented absence of vascular shunts between the systemic and pulmonary circulations, PA blood flow was taken to reflect total body $\mathrm{CO}$. The existence of vascular shunts through either the foramen ovale or ductus arteriosus was excluded by comparison of oxygen contents in blood samples obtained simultaneously from the RA, PA, and aorta. The RA catheter was connected to a pressure transducer to determine central venous pressure (CVP). SVR was determined as the ratio of BP - CVP to CO.

Cerebral Surgery. A modification of the methods of Buckley et al. (11), Gootman (12), Orr et al. (13), Wagerle et al. (14), and Scremin et al. $(15,16)$ was used to measure phasic ICBF. Two separate dissections were made in the right neck region. Through the first dissection, at a level midway between the root of the aorta and the angle of the jaw, the right common carotid artery was identified. The vagus nerve was carefully freed from the vessel at this level, and an external electromagnetic flow probe (EP 406; Carolina Medical Electronics) was placed around the right common carotid artery. A separate dissection was then made at the level of the angle of the jaw, leaving approximately $3 \mathrm{~cm}$ of tissue intact between dissections to isolate the carotid flow probe from physical manipulation during the "mechanical zero" procedure (see below). Through the distal dissection, the right external carotid artery was identified immediately cephalad to the carotid bifurcation and ligated. All residual common carotid blood flow was then taken to reflect unilateral ICBF.

An SS catheter was placed by following the methodology of Park et al. $(17,18)$. A 2 -cm diameter cranial burr hole was made in the midline halfway between the nasion and inion, and the SS was identified. A 16 French plastic catheter (Angiocath; Deseret
Medical, Sandy, UT) was placed in the SS, blood return was verified, and the SS catheter was secured to the scalp. The SS catheter was connected to a pressure transducer for on-line determination of SSP. CPP was determined as the difference (BP - SSP). CVR was assessed as the ratio of CPP to ICBF.

Blood specimens were taken at specified intervals (see below) from the femoral artery, PA, and SS. These samples were analyzed for $\mathrm{pH}, \mathrm{PO}_{2}, \mathrm{PCO}_{2}$, and base excess using a blood gas analyzer (IL 1303; Instrumentation Laboratories, Lexington, $\mathrm{MA}$ ) and for $\mathrm{Hb}$ concentration and oxygen saturation using a co-oximeter (IL 282; Instrumentation Laboratories). The cooximeter had previously been calibrated for piglet blood in our laboratory using a carbon monoxide scrubbing technique (19). From these determinations, QO2, cerebral oxygen delivery, oxygen extraction, $\mathrm{VO} 2$, and $\mathrm{CVO} 2$ were calculated using the standard formulas.

All piglets were allowed to stabilize for approximately $1 \mathrm{~h}$ after surgery before the initiation of any experimental protocol.

Experimental Protocol. Verification of "mechanical zero" signal for ICBF probe. After the ipsilateral external carotid artery was ligated, a mechanically induced "zero flow" ICBF signal was produced by briefly occluding the right common carotid artery through the distal carotid dissection with a vascular clamp (care was taken not to disturb the carotid flow probe that was situated in the proximal carotid dissection). This procedure was performed at frequent intervals throughout each experiment. In addition, at the end of each experiment the accuracy of the mechanical zero procedure was verified by comparing the ICBF flow probe signal during distal occlusion (mechanical zero) with both the signal produced when the probe was turned off (electrical zero) and the flow probe signal after the animal was killed (absolute zero). Verification of the fidelity of the mechanical zero signal is particularly important, inasmuch as it establishes that there is no "offset" or "bias" signal in the determination of ICBF using this flow probe technique (6).

Correlation of ICBF signal with SS blood flow. The blood flow signal from the ICBF probe was calibrated volumetrically against SS effluent using a modification of the methodology of Park $e t$ al. $(17,18)$ and Traystman and Rapela $(20)$. We attempted to ensure that NNLA or INDO administration did not affect the ICBF signal (by, for example, redirecting ICBF to extracranial sites) in the following manner. A mechanical zero flow signal was confirmed, and the value of ICBF determined from the carotid flow probe was noted. The SS catheter was then opened to air, and the volume of blood draining from the SS catheter in $1 \mathrm{~min}$ was measured. Multiple simultaneous comparisons of the ICBF flow signal and SS blood flow were thus obtained both before and after NNLA or INDO administration. In addition, we attempted to preclude the possibility that a release of pressure associated with opening the SS catheter created a factitious surge of SS blood flow by comparing the volume of SS blood draining in the first 30-s interval with the volume drained in the next 30 s. The Pearson product moment correlation of ICBF (dependent variable) with SS flow (independent variable) was determined using the statistical package developed by Glanz (21).

NNLA infusion protocol. After postsurgical stabilization, baseline hemodynamic measurements were determined and blood gas samples were obtained. An i.v. infusion of NNLA $(25 \mathrm{mg} /$ $\mathrm{kg} ; 10 \mathrm{mg} / \mathrm{mL}$ dissolved in $\mathrm{pH}$-adjusted sterile saline; Sigma Chemical Co., St. Louis, MO) was then administered over approximately $20 \mathrm{~min}$ to nine piglets. Hemodynamic measurements were determined continuously throughout the NNLA infusion. Blood samples were obtained at the end of the $25-\mathrm{mg} /$ $\mathrm{kg}$ NNLA infusion.

INDO infusion protocol. After postsurgical stabilization, baseline hemodynamic measurements were determined and blood gas samples were obtained. An intravenous infusion of INDO (5 $\mathrm{mg} / \mathrm{kg} ; 4 \mathrm{mg} / \mathrm{mL}$ dissolved in sterile saline; Sigma Chemicals) was then administered over approximately 20 min to 18 piglets. Hemodynamic measurements were determined continuously 
after initiation of the INDO infusion. Blood samples were obtained at the end of the $5-\mathrm{mg} / \mathrm{kg}$ INDO infusion.

$L A B$ inflation protocol. After postsurgical stabilization, baseline hemodynamic measurements were determined and blood gas samples were obtained. An LA balloon catheter was then inflated in a stepwise fashion in six piglets until $\mathrm{CO}$ and $\mathrm{QO} 2$ had been reduced comparably to the NNLA and INDO protocols. Hemodynamic measurements were determined continuously after initiation of the LAB inflation protocol. Blood samples were obtained at the end of the LAB inflation.

Statistical Analysis. Statistical comparisons were performed both within and across experimental protocols. Within each protocol, the value of each variable postintervention was compared with the value preintervention, using $t$ test for paired samples. Across the experimental protocols, the absolute value of each variable both preintervention and postintervention and the absolute change in each variable during the three interventions were compared using ANOVA. When indicated by ANOVA, pairwise comparisons were subsequently performed using the Student-Neuman-Keuls test. For all comparisons, statistical significance was accepted at the $p<0.05$ level.

\section{RESULTS}

Demonstrating fidelity of mechanical zero procedure for carotid flow probe signal. Figure 1 presents representative tracings of the ICBF signal from a single piglet during the mechanical occlusion zero flow procedure (demonstrating return of the flow signal to its steady state value after producing a mechanical zero), a correlation of the mechanical zero flow signal with electrical zero (produced by turning off the flow probe), and a comparison of the mechanical zero signal during life with the ICBF signal after the animal was killed (absolute zero flow). These observations reveal that there is no offset or bias signal in the determination of ICBF using this flow probe technique, reflecting the fidelity of the mechanical zero flow procedure.

Correlation of flow probe signal with SS effluent during NNLA and INDO infusion. Figure 2 displays simultaneous observations of ICBF (determined from the carotid flow probe signal after ligation of the ipsilateral external carotid artery) and SS flow (determined volumetrically) over a range of CBF during NNLA infusion and INDO infusion in piglets. Two major points are revealed in Figure 2: 1 ) the correlation between CBF assessed by SS flow and CBF assessed by ICBF flow probe signal is excellent, under both NNLA and INDO conditions. The Pearson product moment correlation for ICBF versus SSP during NNLA is $r=$ $0.895(p<0.001)$ and during INDO is $r=0.946(p<0.001)$.

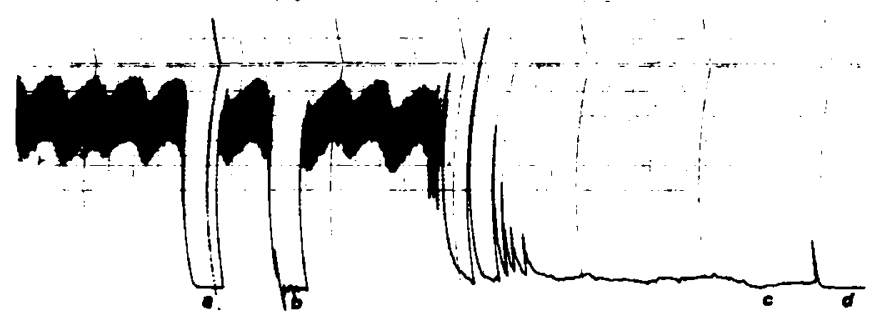

Fig. 1. Correlation of electrical zero, mechanical zero, and absolute zero from a continuous on-line ICBF flow probe signal. $a$, ICBF tracing while the flowmeter is turned off (electrical zero); $b$, ICBF tracing before, during, and after brief occlusion of the distal portion of the common carotid artery after ligation of ipsilateral external carotid circulation (mechanical zero); $c$, ICBF tracing after circulatory arrest produced by euthanasia (absolute zero); and $d$, ICBF tracing while the flowmeter is turned off (electrical zero). Note that the mechanical zero procedure does not affect the steady state value of ICBF and that the noise in the flow probe signal is small compared with the magnitude of the signal. The flow probe signal is identical during electrical zero, mechanical zero, and absolute zero conditions, demonstrating that there is no residual offset or bias ICBF signal using this technique to assess CBF.

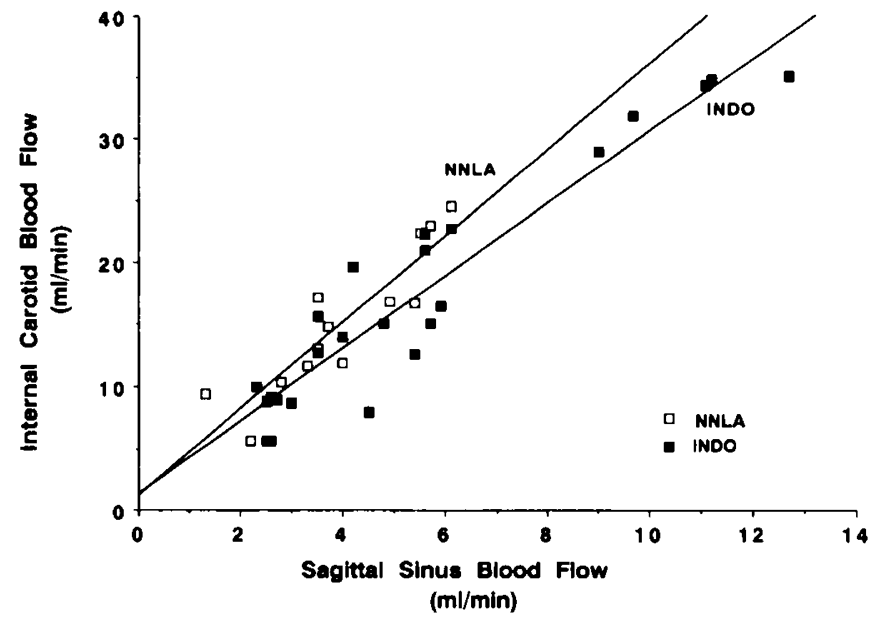

Fig. 2. Correlation of ICBF signal with SS blood flow. Thirty-seven paired observations of ICBF and SS blood flow are displayed during NNLA and INDO infusions. The Pearson product moment correlation for ICBF vs SS blood flow during NNLA is $r=0.895(p<0.001)$ and during INDO is $r=0.946(p<0.001)$. The slopes of the two regression lines do not differ from each other [NNLA $3.48 \pm 0.52$ (SEM) vs INDO $2.93 \pm 0.21$ (SEM); $t=1.14 ; p=$ NS comparing slopes]. The y-intercept values do not differ significantly from each other [NNLA $1.26 \pm 2.21$ (SEM); $1.40 \pm 1.34$ (SEM); $\iota=0.06 ; p=$ NS comparing y-intercepts). The y-intercepts of the regression lines do not differ significantly from zero $(t=0.57$ and 1.04 for NNLA and INDO, respectively; both $p=$ NS). The observation that the regression lines pass through the origin implies that the amount of residual ICBF signal extrapolated to zero SS flow is zero. These data reveal that changes in ICBF parallel precisely changes in SS flow.

The slopes of the two regression lines do not differ from each other [NNLA $3.48 \pm 0.52$ (SEM) versus INDO $2.93 \pm 0.21$ (SEM); $t=1.14 ; p=\mathrm{NS}$ comparing slopes]. 2) The y-intercepts of the regression lines of ICBF versus SS flow do not differ significantly from each other, or significantly from zero, during either NNLA or INDO conditions [y-intercept $=1.26 \pm 2.21$ (SEM) and $1.40 \pm 1.34$ (SEM) for NNLA and INDO, respectively]. These observations reveal that when SS flow is extrapolated to zero ICBF is also zero and demonstrate that changes in ICBF parallel exactly changes in SS flow.

Comparison of hemodynamic effects of NNLA, INDO, and $L A B$ inflation on systemic and cerebral circulations. Figure 3 displays the effects of EDRF inhibition with NNLA, PG inhibition with INDO, and LAB inflation on systemic and cerebral hemodynamics in piglets. Several points are apparent from the figure. The baseline data (preintervention) for each protocol are comparable to each other and to previous observations in piglets reported by us and other investigators using a variety of surgical and anesthetic techniques $(11,22,23)$. Considering the systemic circulation first, each intervention reduced $\mathrm{CO}$ (Fig. $3 A$ ) to an equivalent value $(F=1.23 ; p=\mathrm{NS})$. However, the interventions differed in their effects on BP (Fig. $3 B)(F=78.9 ; p<0.001)$. NNLA and INDO significantly increased BP $(t=5.77$ and $t=$ 7.42 , respectively; both $p<0.001$ ), whereas LAB inflation significantly reduced BP $(t=5.89 ; p<0.001)$. Consequently, SVR (Fig. $3 C$ ) rose significantly and equivalently during both INDO and NNLA infusion compared with LAB inflation $(F=15.28$; $p<0.001)$.

Although $\mathrm{CO}$ was reduced in each protocol, ICBF was affected quite differently by the three intervention protocols (Fig. $3 D$ ). ICBF did not fall significantly during either LAB inflation $(t=$ $1.17 ; p=\mathrm{NS})$ or NNLA infusion $(t=1.58 ; p=\mathrm{NS})$. In contrast, ICBF fell significantly after INDO $(\triangle \mathrm{ICBF}=-8.0 \pm 3.6 \mathrm{~mL} /$ $\min ; t=8.51 ; p<0.001)$. SSP did not change significantly during any protocol and was small $(5.4 \pm 0.8 \mathrm{~mm} \mathrm{Hg})$ compared with $\mathrm{BP}$ in all protocols. Consequently, CPP paralleled BP at all times. 


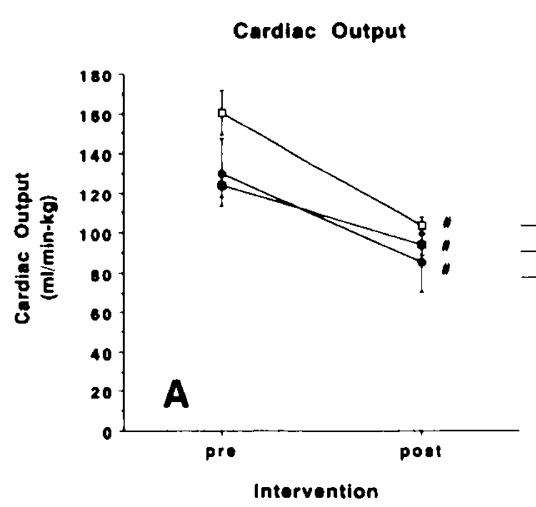

Systemic Vesculer Resistance

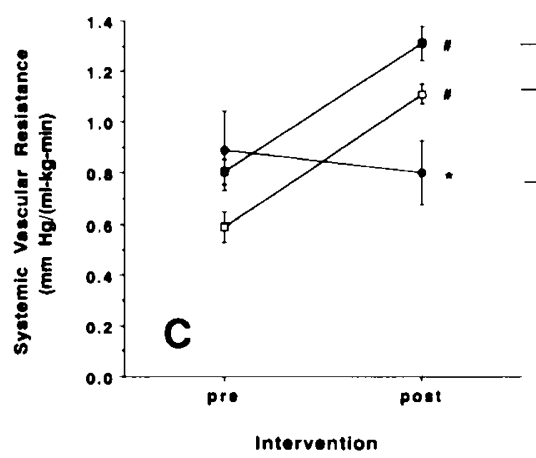

Cerebral Vascular Resistance

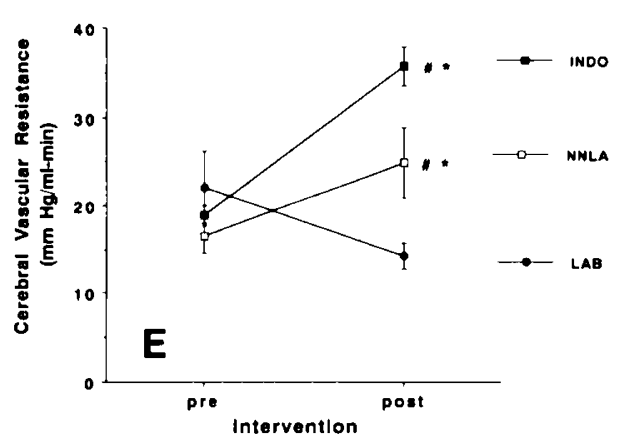

Systemic Blood Pressure

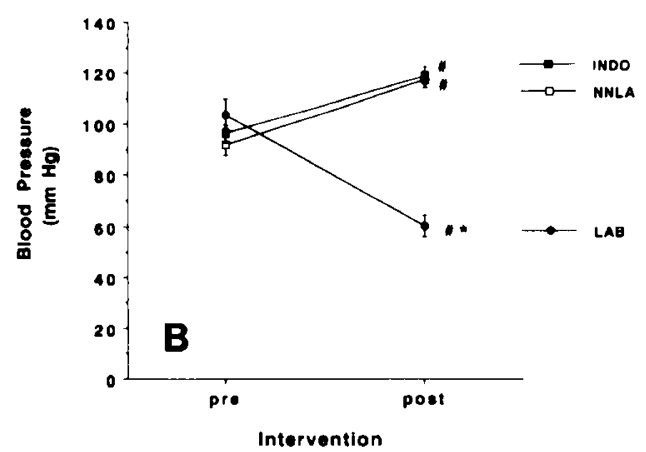

Cerebral Blood Flow

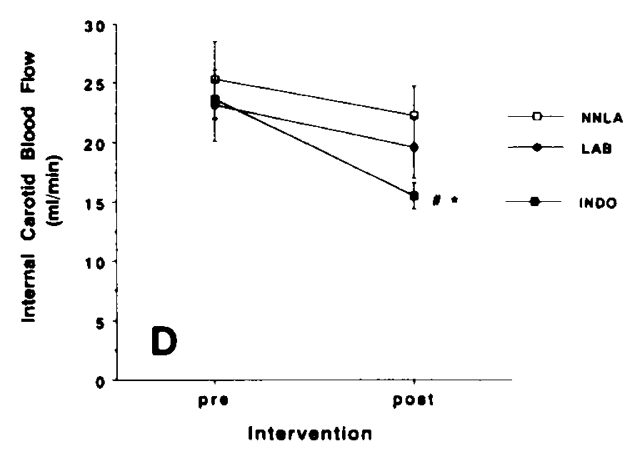

Cerebral/Systemic Blood Flow

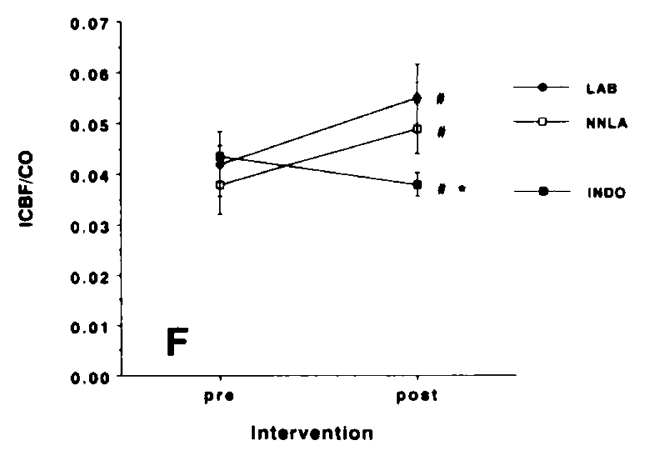

Cerebral/Systemic Vascular Resiatance

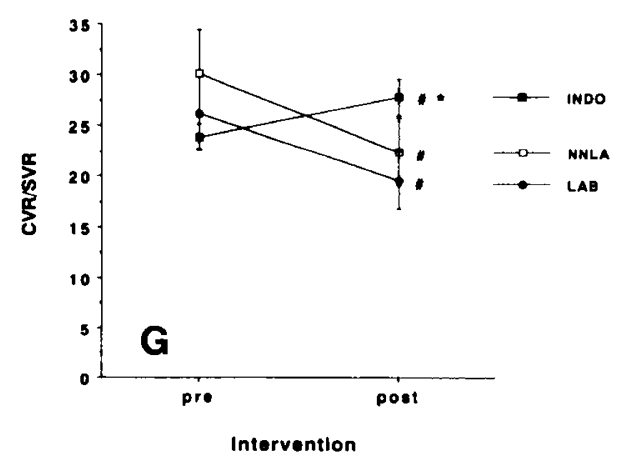

Fig. 3. Comparison of hemodynamic effects of NNLA, INDO, and LAB inflation protocols. Data are displayed as mean \pm SEM. \#, $p<0.05$ preintervention $v s$ postintervention within each protocol (paired $t$ test). ${ }^{*}, p<0.05$ comparing NNLA $v s$ INDO $v s$ LAB protocols (ANOVA). NNLA, INDO, and LAB reduced CO equivalently. NNLA and INDO but not LAB elevated systemic BP and SVR. Despite the equivalent reduction in CO, CBF was preserved during NNLA and LAB protocols, but fell significantly during INDO. The rise in CVR during INDO was significantly larger than the rise during NNLA. Cerebral-specific vascular regulation is displayed in terms of both blood flow (ICBF/CO) and vascular resistance (CVR/ SVR). Despite the equivalent reduction in systemic blood flow during the three experimental protocols, the proportion of systemic blood flow directed toward a unilateral cerebral hemisphere (ICBF/CO) rose significantly during LAB and NNLA but fell significantly during INDO. Similarly, relative CVR (CVR/SVR) fell during LAB and NNLA but rose during INDO. INDO, but not NNLA, abolished the ability of the cerebral circulation to maintain preferential perfusion during a period of reduced systemic blood flow. 
CVR (Fig. $3 E$ ) rose significantly during both the INDO and NNLA protocols $(F=19.4 ; p<0.001)$, with the rise during INDO significantly greater than during NNLA (Student-Neuman-Keuls $q=4.53 ; p<0.05$ ). CVR seemed to fall slightly during LAB inflation ( $t=2.14 ; p=0.08)$.

The ability to monitor simultaneously both the systemic and cerebral circulations allows us to relate changes in one circulation with concurrent changes in the other. The proportion of total systemic blood flow directed to a unilateral cerebral hemisphere is represented algebraically by the fraction ICBF/CO, and changes in this ratio (i.e. $\triangle \mathrm{ICBF} / \mathrm{CO}$ ) operationalize a concept that we refer to as "cerebral-specific" hemodynamic changeschanges that we interpret as what the cerebral circulation is doing that the systemic circulation is not. Furthermore, given the inverse relationship between blood flow and vascular resistance in the systemic and cerebral circulations, cerebral-specific changes can be expressed equivalently in terms of blood flow ( $\triangle \mathrm{ICBF} / \mathrm{CO})$ or vascular resistance $(\Delta \mathrm{CVR} / \mathrm{SVR})$.

Using this formulation, Figure 3 displays cerebral-specific changes in blood flow (ICBF/CO; Fig. $3 F$ ) and vascular resistance (CVR/SVR; Fig. $3 G$ ) during the three experimental protocols. The contrast of these changes during LAB and NNLA protocols compared with INDO is most revealing. During inflation of the LAB catheter, although both CO and ICBF fell, ICBF fell less; consequently, the ratio $\mathrm{ICBF} / \mathrm{CO}$ (the proportion of blood flow directed toward the brain) rose significantly $(t=2.53$; $p<0.05)$ and CVR/SVR fell significantly $(t=2.57 ; p<0.05)$. These findings are hemodynamic expressions of the essential property of the cerebral circulation: preservation of the ability to preferentially direct blood flow toward the brain during periods of reduced systemic flow. Similarly, during NNLA infusion, ICBF/CO also rose significantly $(t=2.42 ; p<0.05)$, and CVR/ SVR also fell significantly $(t=3.79 ; p<0.01)$.

However, after INDO, this essential quality of the cerebral circulation was lost; both $\triangle I C B F / C O$ and $\triangle C V R / S V R$ differed significantly after INDO versus either LAB or NNLA $(F=11.75$ and 15.33 for $\triangle \mathrm{ICBF} / \mathrm{CO}$ and $\Delta \mathrm{CVR} / \mathrm{SVR}$, respectively; both $p$ $<0.001$ ). In fact, the relative proportion of blood flow directed toward the brain was significantly reduced after INDO $(t=3.36$ and 3.05 for $\triangle \mathrm{ICBF} / \mathrm{CO}$ and $\Delta \mathrm{CVR} / \mathrm{SVR}$, respectively; both $p$ $<0.01$ ). These data reveal that, in contrast to inhibition of EDRF with NNLA, which produced significant cerebral vasoconstriction but did not impair the ability of the brain to increase its proportion of systemic blood flow preferentially, inhibition of PG with INDO produced significant cerebral vasoconstriction and did impair the ability of the brain to preferentially direct blood flow toward itself.

Figure 4 displays the effects of NNLA, INDO, and LAB inflation on oxygen consumption comparing the systemic and cerebral circulations. There were no significant differences in the effects of NNLA, INDO, or LAB inflation on VO2, CVO2, or $\mathrm{CVO} 2 / \mathrm{VO} 2(F=1.44, p=0.27 ; F=0.71, p=0.50$; and $F=$ $0.70, p=0.51$, respectively). The protocols did not differ comparing the ability to increase either systemic or cerebral oxygen extraction to preserve metabolism $(F=2.15, p=0.15$; and $F=$ $0.03, p=0.96$, respectively).

For piglets at 1-2 wk of age, we have determined that the average wet weight of the brain is $0.97 \pm 0.11 \%$ of total body weight. Using this value, and assuming that unilateral ICBF represented half of total brain blood flow, the mean value of CBF at baseline was $68.6,73.6$, and $67.2 \mathrm{~mL} / \mathrm{min} / 100 \mathrm{~g}$ of brain weight for the INDO, NNLA, and LAB protocols, respectively ( $F=0.19$ and $p=\mathrm{NS}$, comparing CBF across protocols).

\section{DISCUSSION}

Does ICBF Reflect CBF? We report here the first (to the best of our knowledge) observations of the effects of EDRF inhibition and PG inhibition on the systemic and cerebral circulations in piglets. The import of our observations hinges on the validity of
Systemic Oxygen Consumption

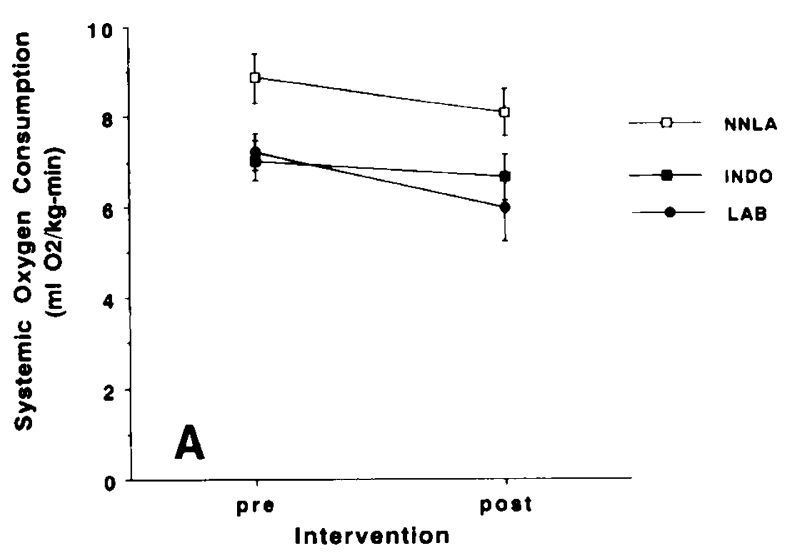

Cerebral Oxygen Consumption

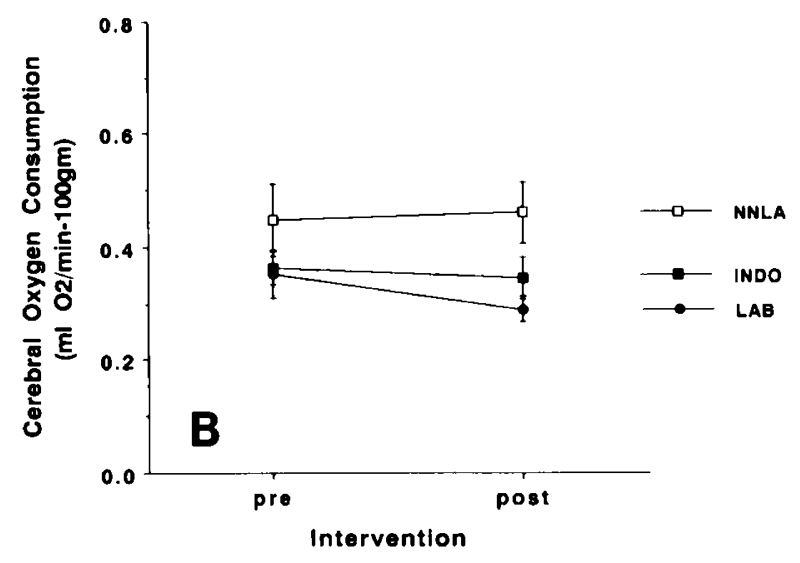

Cerebral/Systemic Oxygen Consumption

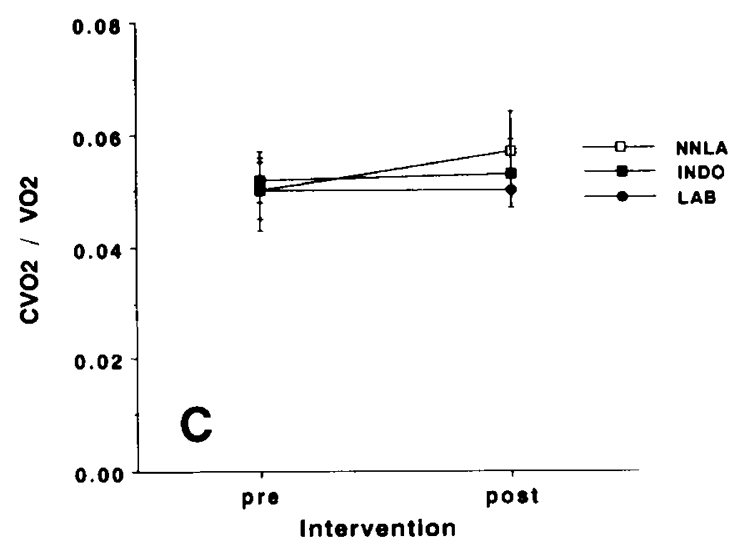

Fig. 4. VO2 and $\mathrm{CVO} 2$ during NNLA, INDO, and LAB protocols. There were no significant changes in $\mathrm{VO} 2, \mathrm{CVO} 2$, or $\mathrm{CVO} 2 / \mathrm{VO} 2$ during the experimental protocols. There were no significant differences in VO2, $\mathrm{CVO} 2$, or $\mathrm{CVO} 2 / \mathrm{VO} 2$ comparing the experimental protocols.

our assessment of CBF. In an attempt to determine both systemic and CBF concurrently and continuously, we chose to measure CBF using an electromagnetic flow probe placed around the common carotid artery in piglets after ligation of the ipsilateral external carotid circulation. This approach to assessing CBF was modified from those previously reported by Scremin et al. (15, 16), Orr et al. (13), Wagerle et al. (14), Buckley et al. (11), and Gootman (12). However, caution must be exercised when using measurements of carotid blood flow (even after ligation of the external carotid artery) to assess cerebral blood flow. In determining the validity of such a technique, two questions must be 
addressed: 1) How reproducible are the data? 2) What exactly is being measured? We take up these two issues in turn.

Figure 1 speaks to the reproducibility of our observations in piglets. Two points can be taken from these data. It is apparent that the magnitude of the flow probe signal is unaffected by assessment of mechanical zero. Indeed, the surgical technique of dissecting the common carotid artery in two separate locations was developed by us for precisely this purpose, i.e. to avoid inadvertent jostling of the flow probe during the mechanical occlusion procedure. Second, Figure 1 reveals that the mechanical zero signal corresponds precisely with the electrical zero and with the absolute zero signal obtained after the animal was killed. The verification of zero flow signals confirms that no offset bias exists in our ICBF measurements and is an important prerequisite for the statistical analysis of changes in ICBF in response to various interventions (e.g. INDO, NNLA, and LAB) (24).

Might flow probe measurements of the internal carotid circulation in piglets, even after ligation of the external carotid circulation, be contaminated with a significant amount of extracerebral blood flow (13)? Alternatively, might administration of INDO or NNLA redistribute internal carotid blood flow to the extracerebral circulation? Figure 2 speaks directly to these concerns. In plotting ICBF versus SS flow, the $y$-intercept can be interpreted as the amount of residual ICBF present when SS flow (taken as a reflection of "true CBF"; see below) is extrapolated to zero; that is, the y-intercept represents residual extracerebral blood flow. As Figure 2 reveals, the y-intercept of the ICBF versus SS flow regression line does not differ significantly from zero and is not significantly affected by administration of either INDO or NNLA. These observations are central to all subsequent interpretation of our data. In each experimental protocol, changes in ICBF parallel precisely changes in SS flow. In other words, to the extent that SS flow reflects overall CBF, the ICBF signal does as well.

Anatomical Correlates of SS Flow and ICBF. Is SS flow an appropriate touchstone with which to correlate ICBF? The venous anatomy of the piglet makes this species particularly well suited to the use of SS flow as a measure of CBF. In piglets, Park et al. $(17,18)$ have demonstrated that 1$)$ jugular venous occlusion produces no discernible effect on SS outflow and 2) occlusion of SS outflow produces a sustained elevation of SSP proximal to the occlusion. These findings fulfill the hemodynamic criteria proposed by Traystman and Rapela (20) and demonstrate that in the piglet [as in the rabbit $(15,16)$ and rat $(25)$ but not the $\operatorname{dog}(20)]$ SS blood is not significantly contaminated with venous blood from extracranial sources. We have replicated these observations in piglets in our laboratory and have previously reported (26) that in piglets the oxygen saturation of SS effluent, but not oxygen saturation of internal jugular venous blood, varies significantly with changes in ICBF induced by hypercarbia, confirming once again that SS effluent, but not internal jugular blood, reflects the cerebral circulation. Finally, we have considered the possibility that a sudden release of downstream pressure associated with opening the SS catheter created a factitious surge of SS blood flow and skewed our measurements. However, when we compared SS flow during the first $30 \mathrm{~s}$ after opening the SS stopcock with SS flow during the second $30 \mathrm{~s}$ of measurement, we found that the two values differed by less than $6 \%$, lending little support to this hypothesis.

The arterial anatomy of the piglet also makes this species particularly well suited for assessing CBF by determining ICBF as we have described here. Unlike the brains of many other species, the brain of the pig relies on the internal carotid arteries for virtually all its blood supply. The vertebral artery system in the pig is almost entirely limited to supplying extracranial structures (in particular, the cervical spinal cord), and the residual branches of the vertebral system that penetrate the foramen magnum to provide blood to the brainstem are small (27). Compression of the vertebral arteries does not change either EEG or intracranial pressure in an isolated perfused pig brain prepa- ration (27). In contrast, occlusion of internal carotid vessels for less than $10 \mathrm{~s}$ produced significant reduction in the amplitude and frequency of EEG signals in the isolated perfused pig brain (27).

The absence of a significant rete mirabile in piglets was another important prerequisite for our decision to use ICBF as a marker of CBF in this species. Were a significant rete mirabile present in piglets (as it is in sheep), the ICBF measurement might be contaminated by postrete redistribution of internal carotid blood flow to the extracerebral circulation (13). The vestigial nature of the rete mirabile in piglets vitiates these concerns in our protocol.

Previously, CBF in pentobarbital-anesthetized piglets has been reported to range at baseline from approximately $35 \mathrm{~mL} / \mathrm{min} /$ $100 \mathrm{~g}$ of brain tissue $(28)$ to $69 \mathrm{~mL} / \mathrm{min} / 100 \mathrm{~g}$ of tissue (29). The steady state values of CBF obtained by us with the carotid flow probe in pentobarbital-anesthetized piglets are at the upper end of this range. These observations suggest that the carotid flow probe assessment of CBF may overestimate absolute CBF. However, considering the fortuitous arterial and venous anatomy of the piglet, the absence of a rete mirabile in this species, the reproducibility of the ICBF signal and the accuracy of assessments of zero flow, the excellent correlation of ICBF with SS flow over a wide range of $\mathrm{CBF}$, and the observation that residual ICBF extrapolated to zero SS flow is zero under all conditions studied, we conclude that in piglets changes in ICBF as measured with a carotid flow probe after ligation of the ipsilateral external carotid circulation can be taken as a reliable reflection of changes in $\mathrm{CBF}$.

What is the Role of EDRF or PG in the "Essential" Property of the Cerebral Circulation of the Piglet? To address this question, we must first consider the role of EDRF and PG in the isolated cerebral circulation of the piglet. We then turn to an examination of the effects of the these compounds on the relationship between the cerebral and systemic circulations.

Role of $P G$ and EDRF in cerebral circulation. The role of PG in the cerebral circulation of piglets has been extensively investigated. Leffler and colleagues (3-8), as well as several other investigative groups $(24,30-32)$, have determined that INDO blocks production of PG in the brain by inhibiting the enzyme cyclooxygenase in the metabolic pathway deriving from arachidonic acid. These authors have shown in pial window preparations that INDO produces cerebral vasoconstriction and have correlated these findings with microsphere measurements, demonstrating a significant reduction in CBF after INDO administration. We have confirmed these observations here. These data suggest that PG participate in basal vascular dilator tone in the cerebral circulation.

EDRF is thought to be identical with the compound NO, formed by the action of the enzyme NO synthase on the substrates L-arginine and molecular oxygen $(33,34)$. EDRF-NO acts in paracrine fashion, diffusing locally from vascular endothelium to vascular smooth muscle, where it increases cytosolic concentrations of cyclic GMP and promotes vascular relaxation (35, 36). The role of EDRF in the systemic and cerebral circulations may be inferred from the effects of inhibiting EDRF production, usually by competitively inhibiting NO synthase with a substituted analog of L-arginine. In intact lambs, Fineman et al. (37) have shown that NNLA competes with L-arginine at the level of NO synthase, causing vasoconstriction in both the systemic and pulmonary circulations. These effects are blocked by pretreatment with L-arginine but not D-arginine. In addition, Fineman et al. have shown that NNLA selectively attenuates the endothelium-dependent vasodilation associated with infusion of acetylcholine and ATP but has no effect on the endothelium-independent actions of isoproterenol and sodium nitroprusside.

Compared with the amount of data on the role of PG, relatively few data exist on the role of EDRF in the cerebral circulation in piglets. Busija et al. (1) demonstrated that L-arginine agonists dilate piglet pial arterioles, an effect that was inhibited by scavengers of $\mathrm{NO}$ ( $\mathrm{Hb}$ and methylene blue) or by N-mono-methyl 
arginine, an L-arginine antagonist related to NNLA. Our findings that EDRF inhibition with NNLA increased CVR and CPP are consistent with the hypothesis that EDRF participates in basal vascular dilator tone in the cerebral circulation.

Relative effects of INDO and NNLA on cerebral vs systemic circulations: operationalizing the concept of an essential organ. Considering the cerebral circulation in isolation, observations of a simultaneous rise in CPP and fall in CBF imply that both NNLA and INDO produced cerebral vasoconstriction. This, we suggest, is certainly a correct description, but according to our formulation, an incomplete one. The ability to monitor simultaneously both the systemic and cerebral circulations allows us to pose an additional question: Given the observation that both INDO and NNLA constrict blood vessels, what are the relative vasoconstrictor effects of these agents on the systemic and cerebral circulations? More specifically, does the brain remain an essential organ-one that is capable of preferentially increasing its proportion of systemic blood flow during a period of reduced $\mathrm{CO}$-after inhibition of either EDRF or PG?

The proportion of total systemic blood flow directed to a unilateral cerebral hemisphere is represented algebraically by the fraction ICBF/CO and changes in this ratio ( $\triangle \mathrm{ICBF} / \mathrm{CO}$ ) operationalize a concept that we have referred to as cerebral-specific hemodynamic change-a mathematical expression of "what the cerebral circulation is doing that the systemic circulation is not." Given the inverse relationship between blood flow and vascular resistance in the systemic and cerebral circulations, cerebralspecific changes can be expressed equivalently in terms of either blood flow $(\triangle \mathrm{ICBF} / \mathrm{CO})$ or vascular resistance ( $\triangle \mathrm{CVR} / \mathrm{SVR})$.

Using this formulation, we have contrasted in the piglet the effects of LAB and NNLA on the cerebral versus the systemic circulation with the effects of INDO. During inflation of the LAB catheter and during NNLA infusion, although both CO and ICBF fell, ICBF fell less. Consequently, in both protocols the ratio ICBF/CO (the relative proportion of blood flow directed toward the brain) rose significantly and the ratio CVR/SVR (the relative resistance of the cerebral circulation vis-à-vis the systemic circulation) fell significantly. These findings are hemodynamic expressions of the essential ability of the brain to preferentially direct blood flow toward itself during periods of reduced systemic flow, even at the expense of the rest of the systemic circulation.

After INDO, however, this essential quality was lost. In contrast to inhibition of EDRF, which produced significant cerebral vasoconstriction but did not impair the ability of the brain to increase preferentially its proportion of systemic blood flow, inhibition of PG produced significant cerebral vasoconstriction and did impair the ability of the brain to increase preferentially its proportion of systemic blood flow during the period of reduced QO2 induced by INDO. These data suggest that PG (inhibited by INDO), but not EDRF (inhibited by NNLA), are critical to the piglet brain's essential property.

Implications of Differential Cerebral Versus Systemic Vasoconstriction during NNLA and INDO Infusion for Conceptions of Cerebral Vascular Regulation. As it is traditionally defined, autoregulation is a pressure-flow phenomenon within an isolated circulation. For example, cerebral autoregulation speaks to the protective ability of the cerebral circulation to maintain CBF (dependent variable) over a wide range of CPP (independent variable) $(30,38,39)$. We have described here an additional aspect of cerebral vascular regulation, along dimensions that might best be characterized as flow-flow. This view speaks to the ability of the cerebral circulation to change relative $\mathrm{CBF}-$ relative, that is, to other organ circulations that are arrayed in parallel with the brain (dependent variable) over a wide range of systemic blood flow (independent variable).

Figure 5 displays observations from the three piglet experimental protocols described here, plotted along normalized pressure-flow dimensions (Fig. $5 A$ ) and normalized flow-flow dimensions (Fig. $5 B$ ). Considering first the pressure-flow dimensions of cerebral vascular regulation (Fig. $5 A$ ), during the $\mathrm{LAB}$ protocol

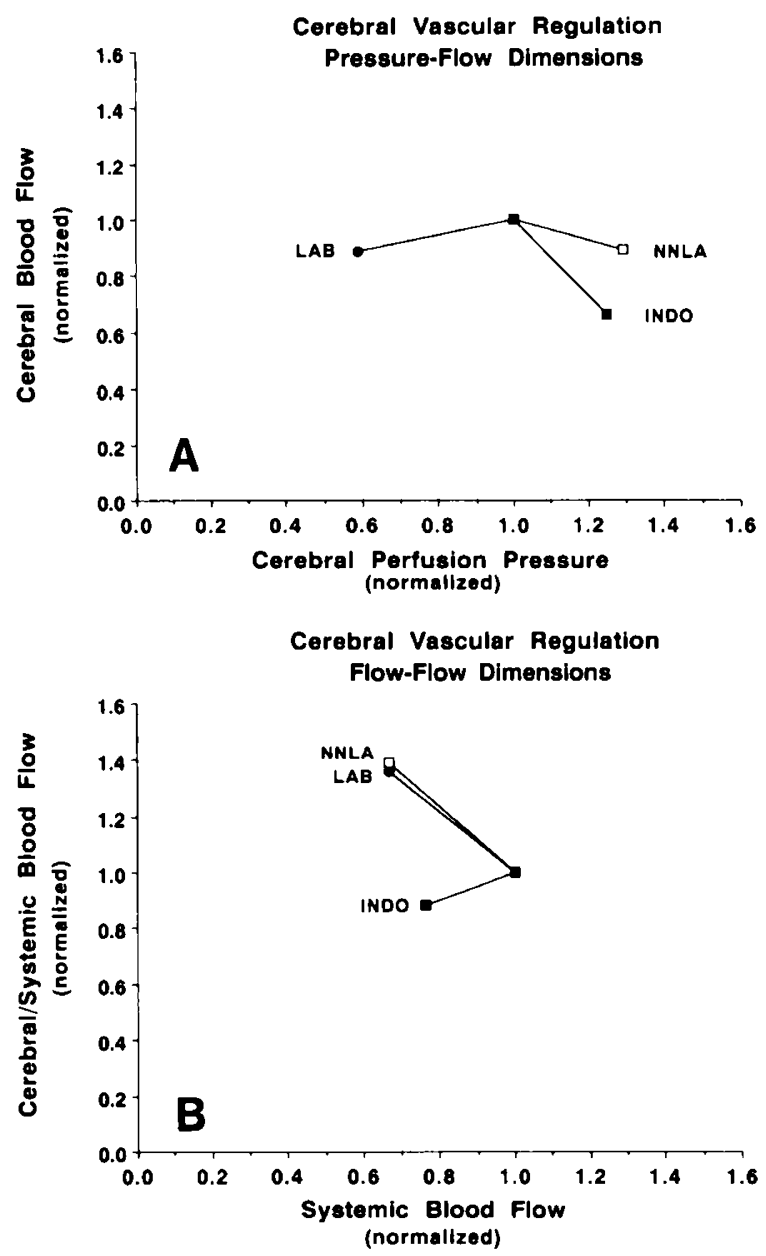

Fig. 5. Cerebral vascular regulation displayed along pressure-flow and flow-flow dimensions. Pressure-flow dimensions $(A)$ display vascular regulation within the cerebral circulation. $\mathrm{CBF}$ was maintained relatively constant as CPP fell during $\mathrm{LAB}$ and was maintained relatively constant as CPP rose during NNLA, reflecting preservation of autoregulation within the cerebral circulation. However, during INDO, as BP rose CBF fell, reflecting a loss of autoregulation within the cerebral circulation. Flow-flow dimensions $(B)$ display vascular regulation in the cerebral circulation vis-à-vis the systemic circulation. Systemic $\mathrm{CO}$ fell equally during the NNLA, INDO, and LAB protocols. During both NNLA and $\mathrm{LAB}$, as $\mathrm{CO}$ fell the relative proportion of systemic blood flow directed toward the cerebral circulation $(\mathrm{CBF} / \mathrm{CO})$ rose, reflecting preservation of the essential status of the cerebral circulation. In contrast, during INDO, as systemic CO fell CBF/CO did not rise, reflecting loss of the essential hemodynamic status of the brain compared with the rest of the body.

CPP fell and CBF was maintained relatively constant within the pressure range examined. Similarly, during NNLA infusion CPP rose and $\mathrm{CBF}$ was maintained relatively constant within the pressure range examined. In contrast, during INDO CPP rose yet CBF fell.

The flow-flow dimensions (Fig. $5 B$ ) of cerebral vascular regulation in piglets compare changes in systemic $\mathrm{CO}$ on the abscissa with simultaneous changes in the proportion of blood flow directed toward the brain (ICBF/CO) on the ordinate. The question specifically addressed by this flow-flow formulation is, given the equivalent reduction in systemic blood flow induced by LAB inflation, NNLA, and INDO (approximately $30 \%$ for each intervention), was the cerebral circulation able to maintain its essential capability, preferentially increasing its share of systemic perfusion, even at the expense of other related, but less essential, organs? As Figure $5 B$ reveals, we found the answer to be yes, yes, and no. During LAB inflation, our control protocol to observe normal physiologic responses of an intact cerebral 
circulation, reduction in $\mathrm{CO}$ by $\sim 30 \%$ was accompanied by a significant $(\sim 40 \%)$ rise in the proportion of blood directed to the brain - the expected response of an essential organ. Inhibition of EDRF, a paracrine mediator that might, a priori, have been thought to be integral to maintaining preferential cerebral perfusion during periods of reduced $\mathrm{CO}$, did not, in fact, impair this process. As CO fell after NNLA, the ratio ICBF/CO rose just as it did after LAB inflation, reflecting the piglet brain's continued ability to extract an increasingly large portion of the dwindling supply of QO2.

In contrast, Figure $5 B$ graphically emphasizes that inhibition of PG synthesis with INDO significantly impaired the ability of the piglet brain to preferentially maintain cerebral perfusion at the expense of systemic perfusion during the period of reduced $\mathrm{CO}$ and $\mathrm{QO} 2$ induced by INDO. After INDO, as $\mathrm{CO}$ fell the fraction $\mathrm{ICBF} / \mathrm{CO}$ did not rise, i.e. the brain no longer acted like an essential organ after INDO, a finding that is readily apparent when cerebral vascular regulation is considered along flow-flow dimensions but is less obvious when vascular regulation is viewed along pressure-flow dimensions.

Under normal physiologic conditions, $\mathrm{CBF}$ is largely independent of systemic blood flow, i.e. $\mathrm{CBF}$ remains relatively constant in the face of many interventions that reduce systemic blood flow (e.g. inflation of an LAB catheter or NNLA administration). Expressing this notion algebraically, for most physiologic interventions that reduce $C O$, the percent $\triangle C B F$ is smaller than the percent $\triangle \mathrm{CO}$; consequently, $\mathrm{CBF} / \mathrm{CO}$ rises. This is the mathematical expression of the widely held view that the brain, like the heart but unlike the gut, is an essential organ. However, under certain conditions, e.g. inhibition of PG by INDO, the piglet brain may lose its cerebral-specific ability to increase $\mathrm{CBF} /$ $\mathrm{CO}$ when $\mathrm{CO}$ falls, i.e. $\mathrm{CBF} / \mathrm{CO}$ may not rise or may actually fall. We suggest that future investigations of the brain specificity of cerebral vasodilation or vasoconstriction, highlighted by consideration of cerebral vascular regulation along dimensions we refer to as flow-flow as well as pressure-flow, are likely to provide new insights into mechanisms and therapies of cerebral vascular disorders.

\section{REFERENCES}

1. Busija DW, Leffler CW, Wagerle LC 1990 Mono-L-arginine-containing compounds dilate piglet pial arterioles via an endothelium-derived relaxing factor-like substance. Circ Res 67:1374-1380

2. Busija DW, Wagerle LC, Pourcyrous M, Leffler CW 1988 Acetylcholine dramatically increases prostanoid synthesis in piglet parietal cortex. Brain Res 439:122-126

3. Leffler CW, Busija DW 1985 Prostanoids in cortical subarachnoid cerebrospinal fluid and pial arterial diameter in newborn pigs. Circ Res 57:689-694

4. Leffler CW, Busija DW 1987 Arachidonic acid metabolites and perinatal cerebral hemodynamics. Semin Perinatol 11:31-42

5. Leffler CW, Busija DW 1987 Prostanoids and pial arteriolar diameter in hypotensive newborn pigs. Am J Physiol 252:H687-H691

6. Leffler CW, Busija DW, Beasley DG 1987 Effect of therapeutic dose of indomethacin on the cerebral circulation of newborn pigs. Pediatr Res 21:188-192

7. Lefler CW, Busija DW, Beasley DG, Fletcher AM, Green RS 1987 Effects of indomethacin on cardiac output distribution in normal and asphyxiated piglets. Prostaglandins 31:183-190

8. Leffler CW, Busija DW, Fletcher AM, Beasley DG, Hessler JR, Green RS 1985 Effects of indomethacin upon cerebral hemodynamics of newborn pigs. Pediatr Res 19:1160-1164

9. Meadow WL, Rudinsky BF, Strates E 1987 Oxygen delivery, oxygen consumption, and metabolic acidosis during GBS sepsis in piglets. Pediatr Res 22:509-512

10. Rudinsky BF, Meadow WL 1992 Relationship between oxygen delivery and metabolic acidosis during sepsis in piglets. Crit Care Med 20:831-839

11. Buckley NM, Gootman PM, Gootman N, Reddy GD, Weaver LC, Crane LA
1976 Age-dependent cardiovascular effects of afferent stimulation in neonatal pigs. Biol Neonate 30:268-279

12. Gootman PM 1985 Cardiovascular regulation in developing swine. In: Tumbleson $M$ (ed) Swine in Animal Research. Plenum Press, New York, pp 1161-1177

13. Orr JA, Wagerle LC, Kiorpes AL, Shirere HW, Friesen BS 1983 Distribution of internal carotid artery blood flow in the pony. Am J Physiol 244: H142H149

14. Wagerle LC, Orr JA, Shirer HW, Kiorpes AL, Fraser DB, DeSoignie RC 1983 Cerebrovascular response to acute decreases in arterial Po, J Cereb Blood Flow Metab 3:507-515

15. Scremin OU, Sonnenschein RR, Rubinstein EH 1982 Cerebrovascular anatomy and blood flow measurements in the rabbit. J Cereb Blood Flow Metab 2:55-66

16. Scremin OU, Sonnenschein RR, Rubinstein EH 1983 Cholinergic cerebral vasodilatation: lack of involvement of cranial parasympathetic nerves. $\mathrm{J}$ Cereb Blood Flow Metab 3:362-368

17. Park TS, Van Wylen DGL, Rubio R, Berne RM 1987 Interstitial fluid adenosine and sagittal sinus blood flow during bicuculline seizures in newborn piglets. J Cereb Blood Flow Metab 7:633-639

18. Park TS, Van Wylen DGL, Rubio R, Berne RM 1988 Brain interstitial adenosine and sagittal sinus blood flow during systemic hypotension in piglets. J Cereb Blood Flow Metab 8:822-828

19. Kirk BW, Raber MB 1973 A practical apparatus for rapid determination of blood oxygen content. J Appl Physiol 34:724-725

20. Traystman RJ, Rapela CE 1975 Effect of sympathetic nerve stimulation on cerebral and cephalic blood flow in dogs. Circ Res 36:620-630

21. Glanz SA 1987 Primer of Biostatistics, 2nd Ed. McGraw Hill, New York

22. Brubakk AM, Oh W, Stonestreet BS 1987 Prolonged hypercarbia in the awake newborn piglet: effect on brain blood flow and cardiac output. Pediatr Res 21:29-33

23. Gopelrud JM, Wagerle LC, Delivoria-Papdopoulos M 1989 Regional cerebral blood flow response during and after acute acute asphyxia in newborn piglets. J Appl Physiol 66:2827-2832

24. Debley VK 1971 Miniature hydraulic occluder for zero blood flow determination. J Appl Physiol 31:138-139

25. Nilsson B, Norberg K. Nordstrom C, Siesjo BK 1975 Influence of hypoxia and hypercapnia on CBF in rats. In: Harper M, Jennet B, Miller D, Rowan J (eds) Blood Flow and Metabolism in the Brain. New York, Churchill Livingstone, pp 9.19-9.23

26. Rudinsky BF, Meadow WL 1991 Internal jugular venous oxygen saturation does not reflect sagittal sinus oxygen saturation in piglets. Biol Neonate 59:322-328

27. Stodkilde-Jorgensen H, Frokiaer J, Kirkeby HJ, Madsen F, Boye N 1985 Preparation of a cerebral perfusion model in the pig: anatomic considerations. In: Tumbleson M (ed) Swine in Animal Research. Plenum Press, New York, pp 719-725

28. Helfaer MA, Kirsch JR, Haun SE, Moore LE, Traystman RJ 1991 Polyethylene glycol-conjugated superoxide dismutase fails to blunt postischemic reactive hyperemia. Am J Physiol 261:H548-H553

29. Wagerle LC, Kumar SP, Belik J, Delivoria-Papadopoulos M 1988 Blood-brain barrier to hydrogen ion during acute metabolic acidosis in piglets. J Appl Physiol 65:776-781

30. Chemtob S, Beharry K, Rex J, Varma DR, Aranda JV 1990 Changes in cerebrovascular prostaglandins and thromboxane as a function of systemic blood pressure. Circ Res 67:674-682

31. DeGiulio PA, Roth RA, Mishra OP, Delivoria-Papadopoulos M, Wagerie LC 1989 Effect of indomethacin on the regulation of cerebral blood flow during respiratory alkalosis in newborn piglets. Pediatr Res 26:593-597

32. Wagerle L.C, Mishra OP 1988 Mechanism of $\mathrm{CO}_{2}$ response in cerebral arteries of the newborn pig: role of phospholipase, cyclooxygenase, and lipoxygenase pathways. Circ Res 62:1019-1026

33. Ignarro LJ 1989 Biologic actions and properties of endothelium-derived nitric oxide formed and released from artery and vein. Circ Res 65:1-21

34. Palmer RMJ, Ashton DS, Moncada S 1988 Vascular endothelial cells synthesize nitric oxide from L-arginine. Nature 333:664-666

35. Furchgott RF 199! Endothelium-dependent relaxation, endothelium-dependent relaxing factor, and photorelaxation of blood vessels. Semin Perinatol 15:11-15

36. Furchgott RF, Vanhoutte PM 1989 Endothelium-derived relaxing and contracting factors. FASEB J 3:2007-2018

37. Fineman JR, Heymann MA, Soifer SJ 1991 N-nitro-L-arginine attenuates endothelium-dependent pulmonary vasodilation in lambs. Am J Physiol 260:H1299-H1306

38. Laptook AR, Stonestreet BS, Oh W 1983 Brain blood flow and oxygen delivery during hemorrhagic hypotension in the piglet. Pediatr Res 17:77-80

39. Leffler CW, Busija DW, Beasley DG, Fletcher AM 1986 Maintenance of cerebral circulation during hemorrhagic hypotension in newborn pigs: role of prostanoids. Circ Res 59:562-567 